Klaus Siebenhaar (Hrsg.)

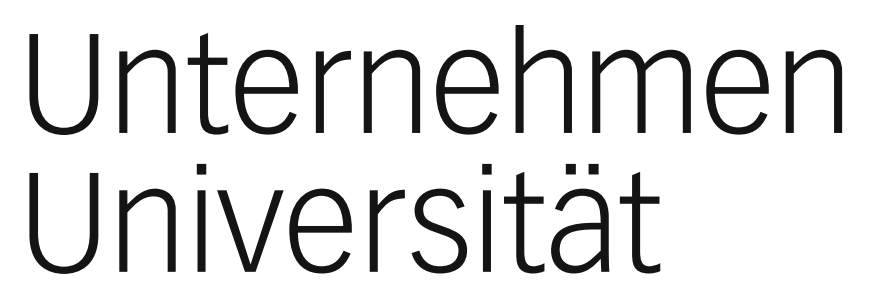

Wissenschaft und Wirtschaft im Dialog

2. Forum Hochschulmarketing der Freien Universität Berlin

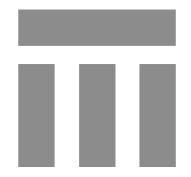


Bibliografische Information der Deutschen Nationalbibliothek

Die Deutsche Nationalbibliothek verzeichnet diese Publikation in der

Deutschen Nationalbibliografie; detaillierte bibliografische Daten sind im Internet über

$<$ http://dnb.d-nb.de> abrufbar.

\section{Auflage 2008}

Alle Rechte vorbehalten

(c) VS Verlag für Sozialwissenschaften | GWV Fachverlage GmbH, Wiesbaden 2008

Lektorat: Katrin Emmerich / Sabine Schöller

VS Verlag für Sozialwissenschaften ist Teil der Fachverlagsgruppe

Springer Science+Business Media.

www.vs-verlag.de

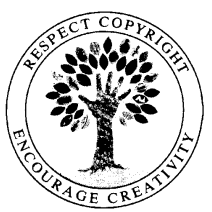

Das Werk einschließlich aller seiner Teile ist urheberrechtlich geschützt. Jede Verwertung außerhalb der engen Grenzen des Urheberrechtsgesetzes ist ohne Zustimmung des Verlags unzulässig und strafbar. Das gilt insbesondere für Vervielfältigungen, Übersetzungen, Mikroverfilmungen und die Einspeicherung und Verarbeitung in elektronischen Systemen.

Die Wiedergabe von Gebrauchsnamen, Handelsnamen, Warenbezeichnungen usw. in diesem Werk berechtigt auch ohne besondere Kennzeichnung nicht zu der Annahme, dass solche Namen im Sinne der Warenzeichen- und Markenschutz-Gesetzgebung als frei zu betrachten wären und daher von jedermann benutzt werden dürften.

Umschlaggestaltung: KünkelLopka Medienentwicklung, Heidelberg

Druck und buchbinderische Verarbeitung: Krips b.v., Meppel

Gedruckt auf säurefreiem und chlorfrei gebleichtem Papier

Printed in the Netherlands

ISBN 978-3-531-16244-7 


\section{Inhalt}

Vorwort

Ursula Lehmkuhl

Begrüßung

Andreas Storm

Gemeinsam für mehr Autonomie und Wettbewerb an den Hochschulen:

Wissenschaft, Wirtschaft und Politik im Dialog

Arend Oetker

Innovationsfaktor Kooperation - neue Wege zu mehr

Austausch zwischen Unternehmen und Hochschulen

Albert Berger

Unternehmen Universität - Universität unternehmen

Klaus Siebenhaar

Lebendige Praxis.

Von der Integration wirtschaftender Unternehmen in Curriculum und

Hochschulmarketing

Stefan Hormuth, Christian Schulze

Die TransMIT Gesellschaft für Technologietransfer mbH -

Flexibilisierung und Optimierung des Technologietransfers

an den mittelhessischen Hochschulen

Holm Keller, Felix C. Seyfarth

Eine Universität erneuert sich grundlegend:

Leuphana Universität Lüneburg

Marcus Beiner

Wissen stiften: Das Beispiel „Pro Geisteswissenschaften“ 
Markus Baumanns

Die Bucerius Law School in Hamburg -

Ein Projekt der ZEIT-Stiftung Ebelin und Gerd Bucerius:

Eine Hochschule als Unternehmen

Henrik Enderlein

Privat finanziert, öffentlich ausgerichtet:

Die Hertie School of Governance als eine besondere Form von „Public-Private-Partnership“

Peter-André Alt, Peter Gaehtgens, Hardy Rudolf Schmitz, René Gurka

Moderation: Armin Himmelrath

Podiumsdiskussion:

Wissenschaft und Wirtschaft zwischen Ökonomisierung und

Autonomieanspruch

Verzeichnis der Autorinnen und Autoren 


\title{
Eine Universität erneuert sich grundlegend: Leuphana Universität Lüneburg
}

\author{
Holm Keller, Felix C. Seyfarth
}

Die Universität in Lüneburg hat innerhalb von 14 Monaten eine grundlegende Reform diskutiert, beschlossen und ihre Umsetzung in Angriff genommen. Ihre Neuausrichtung orientiert die Universität an den Leitideen von Humanismus, Nachhaltigkeit und Handlungsorientierung. Sie ergänzt die klassischen universitären Binnenstrukturen mit klaren Schnittstellen für die Adressaten von universitären Leistungen in Gesellschaft und Praxis. Sie hat ein bundesweit innovatives Studienmodell, das Leuphana College, innerhalb von nur 16 Monaten an den Start gebracht. Ein wesentlicher Bestandteil für den Erfolg dieses Prozesses war und ist die gemeinsame Anstrengung einer Vielzahl interner und externer Beteiligter. Im Folgenden werden Strategien und Maßnahmen des Hochschulmarketings - und damit eines Teilprozesses der Reform in Lüneburg - vorgestellt. Das Papier skizziert einzelne Meilensteine der Lüneburger Neuausrichtung, aus denen sich Kriterien für die Kommunikation öffentlicher Hochschulen in Reformprozessen ableiten lassen.

\section{Zum Begriff des Hochschulmarketings}

Obwohl sich von Greenpeace bis zur Caritas längst eine Vielzahl verschiedener Akteure außerhalb der Wirtschaft aktiver Marketing- und Werbestrategien bedient haben, ist der Begriff des Hochschulmarketing durch seinen Bezug auf den „Markt" in Deutschland immer noch schnell Angriffspunkt für Kritik aus zwei Richtungen. Eine Universität darf, so eine der Auffassungen, mit dem Markt nichts zu tun haben; sie mache sich unseriös, ja verdächtig, wenn sie Angebot und Nachfrage zu Einflussfaktoren ihres Handelns mache. Dieser Strang der Kritik vermutet eine schrittweise Ökonomisierung neoliberaler Prägung - oder etwas schlichter: den Ausverkauf - hinter dem Begriff des Hochschulmarketing und möchte Hochschulkommunikation auf im staatlichen Kontext etablierte Formen von Pressearbeit reduziert wissen. Obwohl die Begriffe des Sozialmarketings und Non-Profit-Marketings bereits etabliert sind, lehnt diese Argumen- 
tation den Gedanken jeglichen universitären Agierens am Markt also grundsätzlich ab.

Ein zweiter Ansatzpunkt der Kritik behauptet, es handele sich bei Hochschulmarketing um eine Kommunikationsform sui generis, die jenseits der normalen Regeln stehe; angesichts einer zunehmend kompetitiven Bildungslandschaft müssten Universitäten zwar gezielt kommunizieren - die gemeinnützigen öffentlichen Einrichtungen gebotene Seriosität und Rücksicht auf besondere interne Beschaffenheiten (Heterogenität, Autonomie einzelner Einheiten) seien jedoch mit Instrumenten wie Corporate Identity, Public Relations und Relationship Management nicht zu verbinden. Damit wird, zehn Jahre nach dem ersten Grundsatzpapier der Hochschulrektorenkonferenz zum Hochschulmarketing, für öffentliche Bildungseinrichtungen ein angeblich nicht den üblichen Gesetzmäßigkeiten unterliegender „Reputationsmarkt" postuliert, der gewöhnlichen, das heißt im weitesten Sinne privatwirtschaftlichen, Instrumenten verschlossen bleibt.

Diese Kritiken sind in sozio-kulturellen Erfahrungen oder historischen Entwicklungen begründet, die an anderer Stelle ausführlich beleuchtet worden sind. Sie tragen jedoch ein verkürztes Bild dessen, was Marketing will, im Hinterkopf: Modernes Marketingverständnis zielt jedenfalls nicht auf Umsatz- oder Gewinnmaximierung, sondern allgemein auf die Kommunikation zur Förderung von Austauschbeziehungen zwischen Individuen oder Organisationen zum gegenseitigen Nutzen (American Marketing Association, 2004). Im Folgenden wird Marketing an Hochschulen dementsprechend umfassend definiert als ein Prozess aus (1) umfassender Bedarfsanalyse bei Zielgruppen, (2) strategischer Ausrichtung der Angebotspalette an identifizierten Bedürfnissen, (3) Aufmerksamkeitsgewinnung für die Alleinstellungsmerkmale, und (4) der Entscheidung für eine Austauschbeziehung zum Erreichen beiderseitiger Ziele. Diese allgemeine Prozessdefinition für öffentliche Hochschulen ist strukturell identisch mit der Prozessdefinition für andere Organisationen.

Aus dieser Definition folgt wesentlich erstens, dass die Instrumente und Mechanismen des Marketings von Unternehmen, anderen Produkten und Dienstleistungen auch für das Hochschulmarketing geprüft werden können und prinzipiell zur Verfügung stehen. Zweitens fällt eine Vielzahl der in Pressestelle, Studienberatung, Außen-/Auslandsamt, Dekanaten und Hochschulleitung angesiedelten Aufgaben unter diese Definition von Hochschulmarketing - häufig ohne, dass sie inhaltlich oder organisatorisch eindeutig trennbar wären. Da diese funktional bereits vorhandenen Prozesskomponenten des Marketings unkontrovers sein dürften, sind Koordination, Strukturierung und Steuerung dieser Teilprozesse deshalb als wichtige Aufgaben des zentralen Hochschulmarketings anzusehen. Es empfiehlt sich dazu ein breit abgestimmtes Vorgehen, das sich 
auf das Erreichen realistischer Ziele konzentriert, anstatt grundsätzliche Legitimationsfragen und Zuständigkeitsbereiche rechtfertigen zu wollen.

\section{Komplexe Angebote, verständliche Formen}

Die Marketingperspektive auf die deutsche Bildungssituation nach Pisa und Bologna lässt sich knapp zusammenfassen: Hier ist ein echter Schildbürgerstreich passiert. Mit der PISA-Studie war die Mär von der Überlegenheit des deutschen Schulwesens als kurzsichtig diagnostiziert - allerdings ohne dass es zu massiven Leistungssteigerungen gekommen wäre. Der sogenannte BolognaProzess hat die im Hochschulwesen bereits bekannten Missstände an manchen Stellen auf eine so gründliche Weise verschlimmbessert, dass sich das ganze Ausmaß des inzwischen vielfach absehbaren Schadens wohl erst in einigen Jahren zeigen wird.

Ursprünglich sollte die Reform zwei Ziele erreichen: Für Studierende und wissenschaftlichen Nachwuchs sollte ein einheitlicher, transparenter und durchlässiger europäischer Bildungsraum geschaffen werden, der einen nahtlosen Ortswechsel zwischen Madrid und Oslo erlaubt. Zweitens sollten in Deutschland als überlang wahrgenommene Studienzeiten, hohes Absolventenalter und hohe Abbrecherzahlen nachhaltig reduziert werden. Nicht zuletzt galt es, die ursprünglich auf kleine Zahlen und eine homogene, kleine Gruppe Studierender ausgelegten Diplom- und Magisterstudiengänge durch einen straffer strukturierten ersten Abschluss, den Bachelor, zu reformieren.

Das Ergebnis kann knapp zehn Jahre nach Bologna noch nicht befriedigen: Weil der Bachelor in Deutschland bisher weder von Personalchefs auf dem Arbeitsmarkt noch von den universitären Akteuren als vollwertiger wissenschaftlicher Abschluss verstanden wird, studieren heute mehr junge Leute länger als vor der Reform - in aller Regel bei gleicher Finanz- und Personalausstattung der Hochschulen. Parallel dazu ermutigt die Exzellenzinitiative des Bundes eine Fokussierung auf Forschungsstrategien bei sträflicher Vernachlässigung der Qualität in der Lehre. Das Lehrangebot fragmentiert in den mittlerweile mehr als 4.000 deutschen Bachelorprogrammen zusehends in verschulte Mikrofächer, befördert den Schnellkonsum lexikalischen Wissens und bietet wenig Forschungserfahrung im Studium.

Die starre Struktur macht selbstgesteuertes Lernen systematisch unmöglich; Wissenserwerb wird im Sinne einer kurzfristigen Arbeitsmarktorientierung instrumentalisiert. Für kleine und mittlere Universitäten in Deutschland bedeutet dies eine ernste Krise, denn ein Scheitern des zweiten Teils der Bologna-Reform - die Einführung der neuen Studienabschlüsse - macht den ersten Teil - die 
Konkurrenzfähigkeit europäischer Hochschulen im Bezug auf Studiengänge oder Nachwuchsförderungen - zu einer unmittelbaren Bedrohung. Für motivierte, begabte, ambitionierte junge Menschen ist die Entscheidung für ein Studium oder eine wissenschaftliche Karriere in England, der Schweiz oder Holland die äußerst naheliegende Alternative zu der Ochsentour durch deutsche Hörsäle.

In der festen Überzeugung, dass der öffentliche Bildungsauftrag auch in Deutschland mit hoher Qualität umsetzbar sein muss, hat die Leuphana Universität Lüneburg einen dreistufigen Neuausrichtungsprozess in Angriff genommen. In der Ausgangssituation war die Universität Lüneburg eine mittlere norddeutsche Universität, die durch strukturelle Unterfinanzierung viele Probleme einer Massenuniversität bewältigen musste. Mit einigen wenigen herausragenden Leuchttürmen war das Gros der Angebote von lokaler, aber nicht überregionaler Attraktivität. Erster Schritt des Reformprozesses war deshalb die langfristige Orientierung der Institution an drei inhaltlichen Leitlinien für ein eindeutiges Profil: Humanismus, Nachhaltigkeit, Handlungsorientierung. In einem zweiten Schritt wurde ein neues Universitätsmodell entwickelt. Alle Fächer und Fakultäten wurden durch eine klare Binnenstruktur in dieses neue Modell integriert. Dem Grundprinzip des „Lebenslangen Lernens" verschrieben, macht das neue Lüneburger Universitätsmodell Angebote für alle Phasen einer Bildungsbiographie vom Abitur über Bachelor, Master und Doktor bis hin zur Fortbildung im Beruf. Darüber hinaus werden verschiedene Schnittstellen gesellschaftlicher Interaktion direkt adressiert. Dieses Modell wurde auf der Basis vielfältiger Reformerfahrungen in der Universität, verschiedener Ideale der Mitglieder der Universität und Kenntnissen ausländischer Hochschulen entwickelt. In der Konzeptionsphase wurde zudem eine Vielzahl von Gesprächen mit externen Wissenschaftlerinnen, aber auch Personalentscheiderinnen und -entscheidern in Unternehmen verschiedener Größe und Ausrichtung geführt.

Den Kern der Universität bilden wenige Forschungszentren mit thematischen Schwerpunkten. Flankiert werden sie von drei „Schools“, die mit verschiedenen Lebensphasen korrespondieren: Das Leuphana College bietet ein einheitliches Studienmodell für alle Studienfächer (Major), ein gemeinsames erstes Semester und ein Fachinhalte ergänzendes und kontrastierendes Komplementärstudium.

Die Graduate School bietet Programme für Graduierten/PostgraduiertenStudiengänge mit akademischem Schwerpunkt, einen an der Praxis orientierten Management-Studiengang und ein entsprechendes Angebot für die Lehrerbildung. Eine Professional School schließlich bildet die Schnittstelle für Weiterbildungsangebote und Transferprojekte, Ansiedlungs- und Gründungsvorhaben in der Region - kurz gesagt: eine Schnittstelle für die Kooperation mit öffentlichen und privaten Institutionen. 
Dieses Modell, seine Umsetzung und Ausgestaltung haben viele Universitätsmitglieder in einem intensiven partizipatorischen Prozess mit mehr als 100 Arbeitsgruppen- und Gremiensitzungen entwickelt. Bei der Einführung des Bachelor-Studiengangs war und ist es das gemeinsame Ziel, einen ernstzunehmenden ersten akademischen Abschluss zu schaffen, der zu lebenslangem Lernen befähigt, wissenschaftliches Arbeiten und methodische Sorgfalt vermittelt, exemplarisch fachliche Vertiefungen zulässt und durch anspruchsvolle Perspektivenwechsel Freiräume des Denkens schafft. Dabei soll Studierenden der Eintritt in die Wissenschaftsgemeinschaft als vollwertige Mitglieder der Universität ermöglicht werden. Sie sollen dadurch ihre Persönlichkeit frei entfalten können und Produzenten, nicht Konsumenten ihrer Studieninhalte sein.

Mit anderen Worten: Für eine gesteigerte Attraktivität der Universität war es vor allem entscheidend, eine große, gemeinsame inhaltliche Grundlage zu finden, die für Studienanfänger, Studierende, wissenschaftlichen Nachwuchs, Verwaltung, Mittelbau und Professorinnen die vielen Einzelleistungen verbinden könnte und die für die externen Stakeholder in Stadt und Region, Wirtschaft und Politik ein schnelles Verständnis komplexer Reformprozesse ermöglichen würde. Die Lüneburger Reform setzt in der grundständigen Lehre an, um so einen wichtigen Beitrag zur Vollendung des Bologna-Prozesses zu leisten. An dieser Stelle, darauf sei für ein besseres Verständnis hingewiesen, steht also die „Marktorientierung“ im Hintergrund, die dem Hochschulmarketing seinen Namen gibt: Die strategische Ausrichtung der Universität orientiert ihre Angebote an Defiziten in der Bildungslandschaft und qualitativen inhaltlichen Anforderungen - statt an vermeintlichen Bedarfen lokaler Unternehmer oder dem status quo hochschulinnerer Gegebenheiten.

\section{Fachexperten und Fachabteilungen arbeiten zusammen}

Sobald der Kern der Reform und die langfristige Zielvorstellung klar geworden sind, können die ersten Schritte einer Kommunikation beginnen. Da mit neuem Wein in alten Schläuchen den inhaltlichen Anliegen nicht gedient ist und sich mit der Fortsetzung etablierter Kommunikationsstrategien nicht die der Reform entsprechende Glaubwürdigkeit erzielen lässt, muss die Veränderung im $\mathrm{Au}-$ Benauftritt mindestens ebenso tiefgreifend und umfassend sein wie die Veränderung im Inneren. Je deutlicher die Botschaft einer klaren Vision, einer idealen Universität, wirklich intensiver Reformarbeit und der Bereitschaft zum Verzicht auf eingeübte Traditionen nach außen getragen wird, desto glaubwürdiger ist der Anspruch auf Besonderheit. Notwendig geworden war deshalb ein grundsätzlich 
neuer Außenauftritt, der in Wertigkeit und Anspruch den Geist der Reform auch für universitätsferne Adressaten sofort erkennbar macht.

Mit universitätseigenen Ressourcen ist eine solche Aufgabe, die ebenso Kriterien langlebiger funktionaler Ästhetik und flexibler Detailumsetzung genügen muss, nicht zu erbringen. Die Universität muss sich eingestehen, dass sie entgegen landläufiger Auffassung nicht alles selber kann, sondern dass ein solches Projekt im vorgesehenen Zeitrahmen jenseits ihrer Kernkompetenzen und Leistungsfähigkeit liegt und von erfahrenen Experten übernommen werden muss. Bei der Suche nach einem professionellen Partner konnte die Hochschulleitung die Agentur Scholz \& Friends in Hamburg gewinnen, die sich aus Begeisterung über das Reformprojekt im Rahmen einer pro-bono-Zusammenarbeit engagiert hat. Während Grundzüge des Außenauftritts verhältnismäßig schnell beschlossen worden waren, stellte die Aufgabe, innerhalb von zehn Monaten Interessenten und geeignete Bewerber für ein bis dato völlig unbekanntes Studienmodell zu gewinnen, die Universität vor eine sportliche Herausforderung. Die beste Idee nützt nichts ohne Bewerber, die auf ihre Qualitäten vertrauen und Teil einer innovativen Hochschulerfahrung werden wollen.

Basierend auf den Unterlagen des Reformprozesses und den Briefings der Hochschulleitung kristallisierte sich eine zentrale Aussage heraus, der die Kerngedanken der Idee in eine für die Adressaten verständliche Sprache übersetzt. Diese Aussage mit Blick auf Studieninteressenten verzichtet auf das Vokabular von methodischer Rigorosität und akademischen Basiskompetenzen. In der Sprache der Zielgruppe sollen vielmehr verunsicherte junge Menschen dazu eingeladen werden zu prüfen, die Lebensphase Studium in einer Gemeinschaft von motivierten, vielseitig engagierten, sozial verantwortungsvollen Kommilitonen zu verbringen. Dafür müssen Erwartungen formuliert werden. Zentrale Aussage wurde: „Der neue Universitätsstandard für eine neue Generation: Anspruchsvoll, offen, vernetzt, lebendig, zukunftsfähig." Diese Aussage ist kein Motto oder Slogan; vielmehr ist er die grundlegende Botschaft für die Arbeit des Hochschulmarketings, die alle weiteren kommunikativen Aktivitäten leitet und bestimmt.

Mit dem neuen Namen „Leuphana“ erinnert die junge Universität an eine wissenschaftliche Urtugend: das Wissen um die Begrenztheit der Erkenntnis. Im „Verzeichnis lateinischer Ortsnamen“ von J. W. Graesse wird die auf dem ptolemäischen Weltatlas aus dem zweiten Jahrhundert verzeichnete norddeutsche Siedlung Leuphana mit dem modernen Lüneburg identifiziert. Ptolemäus stellte das geographische Wissen seiner Zeit mit ca. 6.000 Ortsnamen erstmals in Kartenprojektion zusammen. Nur in Abschriften überliefert, galt das Werk im Mittelalter als Standard - Ptolemäus' Definition der Breitengrade gilt bis heute. Graesses Zuordnung im 19. Jahrhundert ist zweifelhaft - Lüneburg wird erst 
700 Jahre nach Ptolemäus urkundlich erwähnt - und bleibt damit weiterhin Gegenstand wissenschaftlicher Forschung. Aus der Liste möglicher Kandidaten stach der Name unter anderem aus diesem Grund heraus: Statt eines Kunstworts oder eines vielleicht prominenten, aber beliebig wirkenden Namenspatrons bot sich hier eine Bezeichnung als Name an, dem zu ergründende regionale, historische Bezüge zugeschrieben werden, der aber vor allem wegen seiner Seltenheit weder politisch noch touristisch besetzt und somit völlig unverbraucht war. Ein Name, der erst noch zu einer Marke werden kann, sobald er mit assoziativen Bedeutungen aufgeladen wird. Banale, aber in der digitalisierten Welt wichtige Aspekte zeigte eine erste Google Recherche: Eine Suche nach dem Namen ergab mehrere Verweise auf philologische Dokumente mit dem Hinweis auf Lueneburgensis/Leuphana - und sonst nichts.

Weiter wurden ein Logo und eine Bildmarke geschaffen: Der Farbton greift den auf dem zentralen Campus vorherrschenden Rotton der Ziegel auf; die Schrifttype ist seriös, aber nicht altmodisch, modern, aber nicht trendy. Die korrespondierende Farbwelt orientiert sich an den gebrochenen Farben von Kristallen, ein Bezug zu den Salzminen unter der Lüneburger Heide. Die Bildmarke, ein Netzwürfel, erinnert den Betrachter an die trügerischen Eigenheiten der Wahrnehmung: Das Kippbild kann zweidimensional - als Netzwerkknoten oder Salzkristall - betrachtet werden, oder als ein dreidimensionaler auf einer Spitze stehender Würfel, von dem nicht eindeutig zu sagen ist, aus welcher Perspektive er betrachtet wird. Eine fotografische Bildwelt rundet das Corporate Design ab: Die Optik zeigt durchgängig Gruppensituationen und weite Flächen; formgebend ist das Quadrat.

Abbildung 1: Logo und Bildmarke der Leuphana Universität Lüneburg

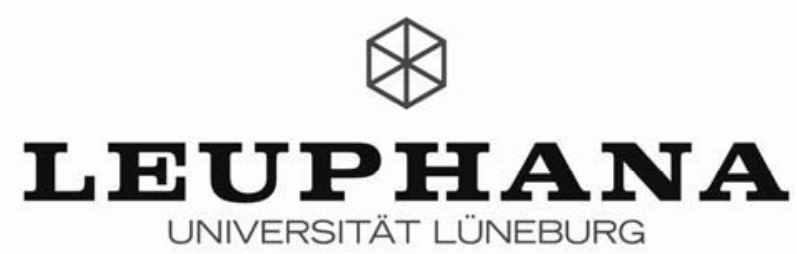


Für externe Zielgruppen können auf dieser Grundlage Drucksorten je nach Anknüpfungspunkt erstellt werden: Informationen über das College für Studienbewerber, eine Beschreibung des Strukturmodells für Kooperationspartner, eine englische Broschüre, in der man das College nicht erklären muss - aber dafür das mittelalterliche Stadtzentrum von Lüneburg. Im Unterschied zur internen Umstellung von Verwaltung, Forschung und Lehre - neue Schriften, Dokumentvorlagen, Visitenkarten, Stempel, Beschilderung, Leitsystem etc. - erfordern die externen Kommunikationsprozesse eine hohe Geschwindigkeit. Der neue Außenauftritt muss haptische Qualität bekommen, greifbare Wirklichkeit werden. Für diesen operativen Teil der breiten Umsetzung müssen interne Ressourcen bereitgestellt werden - die Arbeit der Agentur reduziert sich mit der Übergabe der Designs auf eine Konsultationsrolle. Die Produktion der Inhalte, der roll-out in der Institution, die Dissemination des Materials sowie die Ausgestaltung einzelner Drucksorten sind Teil der Identitätsfindung mit dem neuen Design. Sie brauchen Zeit und stoßen auf verschieden große Akzeptanz.

Der neue Auftritt symbolisiert den Neuanfang nach innen und nach außen. Weil die eigentlichen Reformprozesse langwierig, komplex und in aller Regel unsichtbar stattfinden, hilft das Corporate Design dabei, das neue Selbstverständnis der Institution und seiner Mitglieder zu fördern. Ein qualitativ hochwertiges Design unterstützt eine qualitative hochwertige Wahrnehmung innerhalb und außerhalb der Organisation. Ein neuer Außenauftritt braucht aber Jahre, um selbstverständlicher Teil zu werden, und es vergeht einige Zeit, bis Identifikation und emotionale Nähe wachsen. Wie dies gelingt, hängt von der Organisationskultur und der inhaltlichen Arbeit aller Mitglieder der Universität ab.

\section{Grundsatzbeschlüsse in den Gremien und operative Ausgestaltung}

Ein radikaler Schnitt - in manchen Fällen die gewünschte Außenwirkung einer neuen Corporate Identity - bringt als Kehrseite nach innen zwangsläufig einen schmerzhaften Kulturbruch mit sich. Dieses Phänomen stellt sich unabhängig von allen inhaltlichen Kontingenzen und Kontinuitäten unweigerlich ein: Das Vertraute zu Gunsten des Unbekannten aufzugeben, erhöht die kognitive Spannung und schafft zumindest vorübergehend Unsicherheiten. Im Kontext einer Strukturreform sind solche Verluste besonders belastet - wenn alles in Fluss geraten zu scheint, sind die bewährten Symbole nicht nur aus nostalgischen Gründen wichtige Fixpunkte der eigenen Identität.

In der Tat werden die neuen Symbole - eben weil sie die Veränderung sichtbar machen - intern häufig aufgeladen mit aus dem Reformprozess resultierenden Spannungen, die nun auf die neuen Insignien projiziert werden. Dieser 
Vorgang kann nicht grundsätzlich vermieden, sondern bestenfalls durch gute Vorbereitung und sensible Implementierung gemindert werden, da es sich um die Bedrohung eines natürlichen Sicherheitsbedürfnisses handelt. Die schrittweise Implementierung als institutioneller Lernprozess wird deshalb zwangsläufig Mut, Geduld und Durchhaltevermögen bei denjenigen erfordern, die sich in der Pilotphase engagieren. Sie sind nicht nur Nutzer, sondern auch Multiplikatoren und müssen deswegen gelegentlich Vorwürfe ertragen. Ohne die Bereitschaft einiger, auch ohne Erfolgsgarantie als engagierte Geburtshelfer des Neuen zu wirken, ist ein solcher Prozess im öffentlichen Dienst allerdings nicht vorstellbar.

Skeptiker bezweifeln die Umsetzung einer Corporate Identity an Universitäten generell angesichts der hohen Identifikation von Individuen mit konkret erfahrbaren, dezentralen Einzelbereichen (Instituten, Lehrstühlen, Studiengängen, Professuren) statt mit der als abstrakt wahrgenommenen, diffus konturierten Dachmarke einer Gesamtinstitution. Die nicht-hierarchischen, dezentralen Strukturen einer öffentlichen Hochschule in Forschung und Lehre und die Partizipationskultur an Universitäten erfordern einen geteilten Grundkonsens im ersten Schritt und die nachfolgende interne Umsetzung als opt-in einzelner Bereiche. Die erwünschte identitätsstiftende Markenwirkung kann sich somit nicht, wie in vielen Unternehmen üblich, durch Kontrollversuche oder Sanktionsmaßnahmen entfalten - beides wäre in Universitäten unrealistisch und unangemessen. Sie entsteht allein durch Angebote hochwertiger zentraler Dienstleistungen für dezentrale Forschungs- und Lehreinrichtungen bei der Erstellung von Drucksorten und Webauftritten.

Erfolgsentscheidend ist die richtige Mischung aus Partizipationsprozessen und Exekutiventscheidungen: Ästhetische Entscheidungen und Detailfragen der Ausgestaltung eignen sich nur selten für Lösung im Gremienkonsens. Einzelheiten in der Umsetzung müssen in den dafür einzurichtenden Fachabteilungen vorgenommen werden. Ein konkreter Vorschlag zur Vorlage und Entscheidung in den Gremien muss deshalb, analog zur Arbeit parlamentarischer Ausschüsse, auf dem Weg der Expertenkonsultation vorbereitet werden. Jedoch müssen die für den internen Identifikationsprozess entscheidenden grundsätzlichen Fragen nach Zielen, Anlass, Art und Zweck einer neuen Corporate Identity in der notwendigen Breite und Ausführlichkeit diskutiert und Maßnahmen gemeinsam beschlossen werden. Eine Institution, die ihren Außenauftritt nicht an wesentlichen Stellen trägt, kann nicht von ihm getragen werden. 


\section{Emotionale Erstansprache, sachliche Glaubwürdigkeit}

Der Launch des neuen Modells unter neuem Namen hilft im günstigen Fall, einmalig die gewünschte Aufmerksamkeit für die Reform in der Öffentlichkeit zu generieren. Im Umfeld der Hochschullandschaft genügen eine gute Idee und ein gutes Modell aber nicht (mehr), um neue Gruppen von Studienbewerbern anzusprechen. Eine Studieninnovation entzieht sich zwangsläufig den Multiplikatoreffekten von Alumni oder Rankings, da sie ja erst in Zukunft kommen wird; die Ansprache von für das Studienmodell entsprechend begabten und motivierten Studienanfängern ist somit grundlegend. Erster Schritt, um die überregionale Attraktivität eines Studienangebots, was in Deutschland in großen Teilen regional gewählt wird, für die Zielgruppe zu erhöhen, ist nach der Logik der Informationsgesellschaft die kontinuierliche überregionale Verbreitung der Idee mit dem Ziel, sich als Option im Entscheidungsraum möglicher Bewerbungen zu etablieren. Das mediale Echo des ersten Auftritts allein reicht in Zeiten hoher Ereignisdichte und vielfältiger Studienangebote nicht aus. Kommunikative Herausforderung für das Hochschulmarketing ist die Verankerung der Alleinstellungsmerkmale der Universität im überregionalen Diskurs.

Die Entscheidung für ein Studium und einen Studiengang ist zunächst eine emotionale. Diverse Studien belegen die unverändert schlechte sachliche Information über Studienaufbau und -inhalte. Aus der Vielfalt der möglichen Optionen wählen die meisten Studienanfänger, häufig durch persönliche Empfehlungen gelenkt, weit vor der eigentlichen Bewerbungsentscheidung zunächst einige Universitäten aus, die sie für eine mögliche Bewerbung in Betracht ziehen.

Die Phase rationaler Informationsbeschaffung, in der Prospekte und Informa-tionsblätter gelesen und Informationsveranstaltungen besucht werden, führt letztlich zur Präferenzbildung und zur Studienplatzentscheidung. Um in dieser Phase noch $\mathrm{zu}$ den Kandidaten $\mathrm{zu}$ gehören, muss eine Universität ihre Leistungs- und Alleinstellungsmerkmale früh in den Köpfen von Gymnasialdirektoren, Eltern, Bildungspolitikern, Journalisten und Mitschülern verankern. Die noch im Reformprozess stattfindende Kommunikation an eine Zielgruppe, die sich mit einer Vielzahl sachlicher Einzelheiten ausführlich befassen muss, kann deshalb in der Frühphase konsequent auf emotionale Botschaften setzen.

In ihrer narrativen Form unverändert muss diese Botschaft über alle Kanäle hinweg immer wieder neu erzählt werden, in Variationen zwar, aber stets stringent orientiert an der Leitidee. Ausgehend von einer realistischen Analyse der rationalen Kriterien, die bei der Entscheidung für eine Universität in die Waagschale geworfen werden - geographische Nähe, Stadt-/Universitätsgröße, Fachstudium - sind maßgebliche Parameter für eine emotional aufgeladene Botschaft, ihre Authentizität und ihre Konsistenz. 
Die häufigen Berührungsängste mit Hochschulmarketing resultieren aus einer schnellen Gleichsetzung von Marketing mit Werbung - letzteres ist aber nur ein Marketingwerkzeug, das im öffentlichen Bereich in aller Regel schon aus Kostengründen in nicht nennenswertem Maße zum Einsatz kommt. Stattdessen kann Hochschulmarketing bemerkenswerte Effekte durch die konsequente emotionale Ergänzung universitärer Kommunikation erzielen, indem sie Charakteristika der Universität - respektvolle Gemeinschaft der Lernenden, Verantwortungsübernahme im geschützten Umfeld, Motivation für soziales Engagement - in den Vordergrund stellt.

Eine fundamentale Rolle bei diesem Prozess spielt der Webauftritt der Universität. Die Website ist für viele Studieninteressierte (und andere) der erste intensive und bei entsprechender Entfernung einzige visuelle Kontakt mit der Institution. Das Missverhältnis aus einem Überangebot an heterogenen Inhalten und knapper Ressourcenausstattung in Pressestellen und Rechenzentren führt leider immer noch häufig zu Webgestaltungen, die an elektronische Vorlesungsverzeichnisse erinnern. Selbst hochwertige Designs konzentrieren sich gemäß dem rational-sachlichen Diskurs der Wissenschaft auch online auf Sachinformation und eine hierarchische, verwaltungstechnische Sicht auf die Institution - für Außenstehende entsteht häufig ein verwirrender Eindruck. Die sorgfältig entwickelte sozio-emotionale Dimension einer Universitätsmarke fällt hier häufig technischen, pragmatischen oder finanziellen Begrenzungen zum Opfer. Die holistisch-emotionale Ansprache für die Besucher der Website ist von nicht zu unterschätzender Bedeutung. In Lüneburg ist deshalb auch auf den typischen Portalcharakter einer Universitätshomepage verzichtet worden - zu Gunsten einer klaren, übersichtlichen Gliederung. Der Einsatz von Bildern transportiert den Gemeinschaftsgedanken, das Miteinanderlernen und die Atmosphäre der Campusuniversität. Die Glaubwürdigkeit der Leitidee spiegelt sich hier auch auf der Meta-Ebene verwendeter Inhalte wieder: Podcasts, Newsfeeds, Interaktion und Kommentarfunktion sind Web-2.0 Technologien ${ }^{1}$, die einen inhaltlichen Anspruch auf Innovation unterstreichen.

Aus Kostengründen bietet es sich dabei an, das Internet konsequent als Leitmedium für die Außenkommunikation zu verwenden und andere Drucksorten online verfügbar zu machen, sie aber am Primat der Online-Inhalte auszurichten. Schließlich dürfen trotz der aktiven Betonung emotionaler Aspekte sachlich-seriöse Informationsinhalte nicht zu kurz kommen. Damit ist die Integration von Drucksorten in die Website als PDF-Datei oder e-Papier ebenso ge-

\footnotetext{
${ }^{1}$ Als Beispiel sei die für das Leuphana College eingesetzte Web-Applikation „Studienwunsch College“ genannt, die es Bewerbern ermöglichte, ein kurzes eigenes Profil samt Foto in einer „Bewerbergalerie“ anzulegen. In kürzester Zeit entstand auf der Website eine sichtbare community mehrerer hundert künftiger Bewerber mit hohen wiederkehrenden Besucherzahlen.
} 
meint wie die Möglichkeit, universitäre Veranstaltungen online anzukündigen und zu dokumentieren. Eine auf diese Weise erreichte Bindung der Nutzer an das Online-Angebot leistet einen wertvollen Beitrag für die soziale Gestaltung der Markenidentität.

Der Wirkungsgrad der so eingesetzten Ressourcen ist schwierig zu kontrollieren. Naheliegende Messgrößen sind die Zahl der Bewerber und die Annahmequote unter den zugelassenen Bewerbern - die allerdings durch mehrere Einflussfaktoren bedingt werden und somit nur eingeschränkt operationalisierbar sind.

Die Bewerberquote hat sich mit der Einführung des neuen Modells im Wintersemester in Lüneburg im Vergleich zum Vorjahr leicht erhöht. Angesichts der auslaufenden bekannten Studiengänge einerseits und der parallel (unabhängig vom Lüneburger Reformprozess) neu anfallenden Studienbeiträge war in Niedersachsen ein Rückgang der Zahl von Studienbewerbern erwartet worden. Dass dieser Rückgang in Lüneburg ausblieb, kann als ein vorsichtiger Erfolg für die gelungene Vermittlung der Qualitäten des Studienmodells gewertet werden. Aussagekräftiger ist hingegen die Annahmequote unter den im Leuphana College zugelassenen Bewerbern. Durch die Möglichkeit, sich ohne nennenswerten Zeitaufwand und ohne Kosten für mehrere Studienplätze zu bewerben, sind Bewerbungen an mehreren Universitäten keine Seltenheit.

In den vergangenen Jahren lag die Annahmequote der Lüneburger Universität - also die Zahl der nach Zulassung tatsächlich immatrikulierten Erstsemester - bei rund $30 \%$, ein Anzeichen dafür, dass viele Bewerber einem Studienplatz in Lüneburg das Angebot anderer Standorte vorgezogen haben. Die Annahmequote im Wintersemester 2007/08 für das Leuphana College lag hingegen bei $47 \%$ - eine erfreuliche Entwicklung, die nebenbei auch der Hochschulverwaltung zu Gute kommt, weil sie die Planbarkeit erhöht und die Nachrückerbetreuung vermindert.

\section{Eindeutiges Branding, flexible Markenführung}

Universitäre Strukturen sind typischerweise geprägt von hoher Heterogenität. Bestimmte Einrichtungen und Teilbereiche verfügen über Teilautonomien; eine Vielzahl dezentraler Prozesse entzieht sich der Steuerung durch eine vergleichsweise schwache Leitungsebene, die Einheiten arbeiten autonom. In der Kommunikation nach außen dominieren Vielfalt und Uneinheitlichkeit. Im Gegensatz zur klassischen Unternehmenskommunikation geht deshalb ein großer Teil der Energie von Pressestellen und Öffentlichkeitsarbeit nach innen, und zwar mit dem Ziel einer stärkeren universitären Integration, die Voraussetzung 
für eine erfolgreiche Positionierung der Universität in der wissenschaftlichen wie größeren Öffentlichkeit und eine Markenführung ist.

Die grundsätzliche Schwierigkeit bei der Entwicklung gemeinsamer Zielsysteme der ganzen Hochschule spiegelt sich auch in der Ausgestaltung der Corporate Identity wider. Alle Elemente des Designs - sowohl im Druckbereich als auch online - müssen deshalb modulare Verwendung in dezentralen Kontexten zulassen. Eine Universität kann nicht in einem strengen Logikbaum kategorisiert werden; das Branding muss - soweit dies leistbar ist - deshalb interne Heterogenität, Zielkonflikte und Widersprüchlichkeiten berücksichtigen, auffangen und produktiv machen. Corporate Identity wird sich deshalb immer nur langsam und langfristig entwickeln und muss die Grundprinzipien der akademischen Denkweise - Pluralismus, Freiheit und Disput - anerkennen, ohne völlig unkenntlich zu werden.

Für eine erfolgreiche Branding-Strategie müssen deshalb auf drei Ebenen Antworten gefunden werden, die universitären Realitäten gerecht werden. (1) Eine gezielte Branding-Strategie muss klar kennzeichnen, an welchen Stellen autorisierte, offizielle Kommunikation der Hochschule als übergeordneter Institution stattfindet und wo Kommunikation aus den einzelnen, dezentralen Einrichtungen heraus oder von Dritten passiert. Konsistenz in der Darreichung von Nachrichten ist für Unterstützer und Interessenten ein wichtiges Thema und muss entsprechend behandelt werden. (2) Die Branding-Strategie muss den Teilidentitäten von Fakultäten, Instituten, Bereichen, Einrichtungen etc. in soweit Raum geben, dass ein widerspruchsfreies Nebeneinander als Teil der Identität der Hochschule möglich wird. Ein Weg, um dies zu erreichen, sind Sublogos und Bereichslogos in Anlehnung an die Dachmarke. (3) Eine nachhaltige Branding-Strategie akzeptiert und unterstützt auch kritische und satirische Kommunikation, die sich auf die Dachmarke bezieht oder sie benutzt. Wird eine Hochschulmarke nicht als monolithischer Block, sondern als Assoziationswolke verschiedener Teilöffentlichkeiten verstanden, dann zahlt der long tail verschiedener Markenaspekte langfristig in das Profil der Marke ein.

\section{Public Relations als Hauptinstrument}

Die gewöhnlichen Aktionsparameter des Marketings stehen Hochschulen in der Tat nur eingeschränkt zur Verfügung. Werbemaßnahmen, Preisanreize und Point-of-Sale Marketing kommen in Deutschland zumindest nicht in nennenswerten Größenordnungen zum Tragen; nicht nur aus Kostengründen sind zumindest im Bereich der Studierendengewinnung andere Maßnahmen auch zu 
bevorzugen. Distribution schließlich scheidet für die überschaubare Zukunft ebenfalls aus - die Wissenschaft bleibt ortsgebunden.

Umso ernster muss das verbleibende Instrument Public Relations genommen werden. Eine professionelle Presseabteilung muss Beziehungen zu Journalisten aufbauen und universitäre Aktivitäten aktiv kommunizieren, um im Gespräch zu bleiben oder ins Gespräch zu kommen. Das bedeutet, dass die Universität ihren qualitativen Anspruch und ihre inhaltlichen Leitlinien mit Projekten und Personen konkretisieren muss, um die Ernsthaftigkeit ihres Anliegens zu verdeutlichen. Persönlichkeiten, die sich als Botschafter eine Vision zu eigen machen, werden sie weitererzählen und mit ihr identifiziert werden. Die Universität muss sich auf ihre Stärke besinnen, durch Ideen und Erkenntnis zu überzeugen. Vier ganz unterschiedliche Beispiele für dieses Arbeiten seien genannt:

1. Gemeinsam mit den Universitäten Mannheim, Trier und Essen kooperiert die Leuphana Universität Lüneburg mit der Jimmy \& Rosalynn CarterStiftung, die Kooperationsinitiativen zwischen Hochschulen und Gemeinden fördert.

Die Botschaft des Friedensnobelpreisträgers Carter, dass aus dem Privileg der Bildung eine Verantwortung für persönliches Engagement in nächster Nähe erwächst, ist ein fester Bestandteil der das College tragenden Überlegungen. Im Rahmen der Preisverleihung der Stiftung 2007 in Lüneburg hat Präsident Carter die Erstsemester in bewegenden Worten an ihre Verantwortung erinnert und ihren Mut begrüßt, sich auf Unbekanntes einzulassen. Solche Partnerschaften mit universitätsfernen Verantwortungsträgern des öffentlichen Lebens sind für die lebensweltliche Verankerung akademischer Lehre elementar, und sie tragen zur öffentlichen Wahrnehmung der Universität als zivilgesellschaftlicher Akteurin wesentlich bei.

2. Im Rahmen der Startwoche des Leuphana College haben alle Erstsemester eine groß angelegte Fallstudie zur Sanierung eines fiktiven öffentlichen Theaters bearbeitet - und zwar innerhalb und außerhalb des Campus. Die Universität hält damit nicht nur vom ersten Tage an ihr Versprechen ein, ein interdisziplinäres, problembezogenes Studium zu bieten, sondern sie sendet auch ein deutliches Signal der Öffnung in die sie umgebende Kommune. Medien, Politiker, Gewerkschaften, Theaterleute sind als Rollenspieler eine Woche lang Teil einer fiktiven Welt geworden, um eine möglichst lebendige Situation herzustellen. Die Leuphana verdeutlicht, dass Universitätsmitglieder nicht nur Mitglieder der scientific community sind, sondern auch Bürger einer Stadt, und heißt die Studierenden in beiden Gemeinschaften willkommen. 
3. Im Rahmen der Ziel-1-Förderung in Niedersachsen hat die Universität einen EU-Antrag gestellt, um ihrer Verantwortung auch auf regionaler Ebene gerecht zu werden. Das Selbstverständnis eines aktiven, gesellschaftlich engagierten Partners für die regionale Entwicklung stellt sich dem klassischen Bild des akademischen Elfenbeinturmes bewusst entgegen. Die besonderen Qualitäten einer Hochschule - internationale Kontakte, Nähe zum aktuellen Forschungsstand, skalierbare Infrastruktur, Absolventenzyklen - konsequent auch als Standortfaktor in der Region zu betrachten, untermauert die auf langfristige Stabilität gerichteten Aspekte der Reform.

4. Die Entwicklung des Universitätscampus speist sich aus der Einsicht, dass Ästhetik und Kreativität wichtige Komponenten von Lernprozessen darstellen. Im Rahmen einer fakultätsübergreifenden Projektwoche in der vorlesungsfreien Zeit haben Studierende aller Fachrichtungen die baulichen, energetischen, ökologischen und informationellen Aspekte des Campus analysiert und dokumentiert.

Solche dem traditionellen universitären Selbstverständnis eher fernen Projekte sind Teil einer Reform, die Forschung und Lehre im Rahmen einer gesellschaftlich fest verankerten Universität begreift. Die Universität als öffentliche Einrichtung tritt dazu in einen partnerschaftlichen Dialog mit der sie umgebenden Zivilgesellschaft. Die äußere Wahrnehmung der Universität als Akteurin von unmittelbarer sozialer Relevanz eröffnet der Institution die Chance, ein neues Modell für den Bildungsstandort Deutschland medienübergreifend vorzustellen. Damit sind public relations im ursprünglichen Sinne gemeint: Über die Kommunikation von Forschungsergebnissen und Lehrveranstaltungen hinaus nimmt die Universität ihre Rolle als Gesprächspartner für die komplexen sozialen Fragen der Zukunft wahr: Wenn sie sich ernsthaften Fragen ernsthaft widmet, wird ihr öffentliche Aufmerksamkeit sicher sein.

\section{Erfolgsfaktoren: Effizientes Marketing in der Hochschule}

Universitäre Kommunikationsstrukturen sind typischerweise gekennzeichnet durch eine im Vergleich zum privaten Sektor relativ hohe Heterogenität, Komplexität, Autonomie und Partizipation. Erfolgreiche Markenbildung öffentlicher Universitäten muss damit gleichzeitig allgemeine Prinzipien der Markenbildung und spezifische Prozesse der Hochschulentwicklung und -kommunikation meistern. Darüber hinaus werden Reformprozesse von manchen auch als implizite Kritik am Bestand wahrgenommen. Das Beispiel der Leuphana Universität 
Lüneburg zeigt allgemeine Strukturen und kontextspezifische Pfadabhängigkeiten für einen solchen Prozess. Identifizierbare Erfolgsfaktoren sind (a) der organische Bezug kommunikativer Inhalte auf die lokale Situation, (b) die intensive Betreuung des Prozesses durch externe Partner und zusätzliche Ressourcen außerhalb bestehender Organisationseinheiten und (c) die zügige Projektumsetzung innerhalb eines begrenzten Zeithorizontes. 